\title{
External recording of gastric activity: The electrogastrogram*
}

\author{
JOHN K. STEVENS \\ Pennsylvania Stote University, University Park, Pennsylvania 16802 \\ and \\ NORMAN WORRALL \\ University of London Institu te of Education, London, England
}

\begin{abstract}
Reports in the American and Russian literature have suggested that recording of electrical activity from electrodes on the surface of the abdomen (electrogastrogram, or EGG) might be used as a means of measuring gastric motility for psychophysiological research and for clinical purposes. However, the complexity of the recordings has presented problems in specifying relations between the EGG and other measures of gastric activity. In this study, cats are used to demonstrate the validity of the EGG as a measure of gastric activity by taking recordings from a strain gauge on the stomach wall simultaneously with electrical recordings from the surface of the abdomen. The present report also demonstrates that autocorrelation and Fourier analysis may be used to reduce record complexity and to provide information unattainable by conventional methods.
\end{abstract}

External surface recording of gastric motility is among the least used of psychophysiological measures. The clinical applications of an external recording method for patients with gastrointestinal disorders would seem self-evident, but also experimental studies of problems as wide ranging as motion sickness and feeding behavior would suggest a place for a simple method of recording gastric activity, if only as an ancillary measure. This lack of widespread use exists in spite of the evidence on the electrogastrogram (EGG) in Russell and Stern (1967). However, it is in Russell and Stern's chapter that we may discover at least part of the reason why this indicator has failed to receive widespread acceptance. This is because it is not known how the electrical record is related to the mechanical activity it purports to measure.

Research on the functioning of the gastrointestinal tract has employed a variety of methods for recording and analyzing gastric motility. Most of the methods, i.e., recording intragastric pressure, fluoroscopy, electromagnetic, and telemetering techniques, require placement of a recording device or one of its components in the stomach and thus involve the risk of producing artifacts in normal activity by direct stimulation of receptors in the stomach wall. The possibility that the dynamics of gastric motility might be studied in terms of changes in bioelectric potentials generated by the stomach and detected at a distance

\footnotetext{
*This research was carried out while both authors were at Indiana University, and supported by Office of Naval Research Contract Nonr 908-15. Thanks are due to R. W. Russell, C. D. Barnes, J. Furedy, K. Kveim, and E. Helmer for help and advice. Silicone rubber materials were kindly provided by the Dow Corning Center for Aid to Medical Research, and X-ray facilities were provided by courtesy of the Indiana University Student Health Center. Requests for reprints should be sent to Norman Worrall, University of Lond on Institu te of Education, Malet St., London WC $1 \mathrm{H}$ OAA, England.
}

from their source was recognized by Alvarez as long ago as 1922. From electrodes positioned on the skin surface over the stomach, Alvarez recorded a predominant activity at three deflections per minute. With the development of more sophisticated apparatus for amplifying bioelectric potentials, Soviet workers turned their attention to applications of the method and now have it in experimental use for detection of gastric disorder (Krasil'nikov, 1964). In addition, Goodman et al (1964) in the United States have developed a method of electrogastrography employing intragastric electrodes. His method has proved to be very helpful in diagnosis of specific gastric disorders.

During the period between the Alvarez report and today's clinical applications, research effort has been directed toward three basic methodological issues: standardizing the recording techniques for the EGG, refining the methods of analyzing the records obtained, and establishing the EGG as a valid clinical and research tool (see Russell \& Stern, 1967). It was suggested above that one reason for the failure of the EGG to achieve wider application in psychophysiological research was a need to show the relationship between the electrical record and underlying mechanical activity, so that proper interpretation could be made. The studies reported below were addressed to this problem. Using the cat, significant relations were found between mechanical activity, as recorded from force transducers located directly on the outside wall of the stomach and electrical activity recorded via electrodes placed on the skin of the abdomen using the Davis technique (Davis, Garafolo, \& Gault, 1957). The recordings also provided material for demonstrating that computer methods of autocorrelation, crosscorrelation, and Fournier analysis could be a less laborious approach to the quantitative analysis of the EGG. 


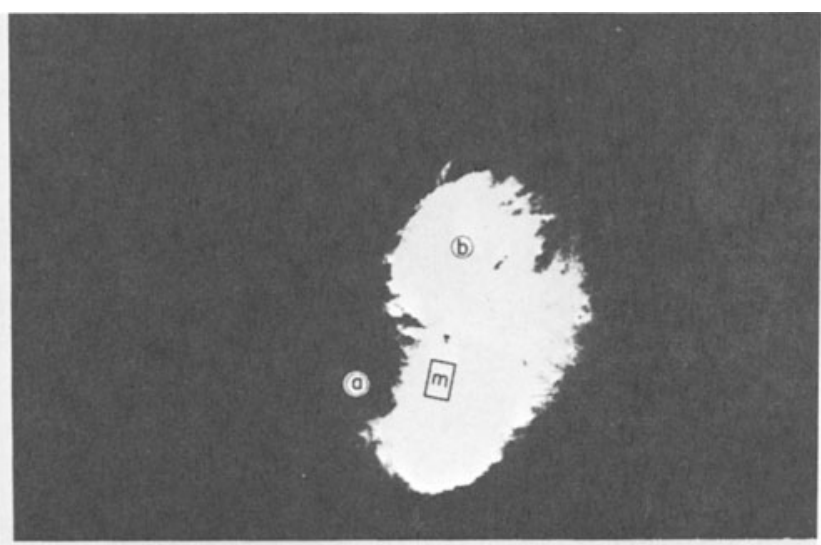

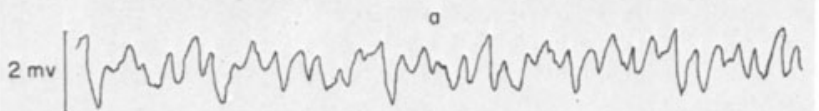

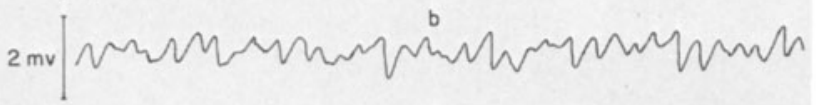

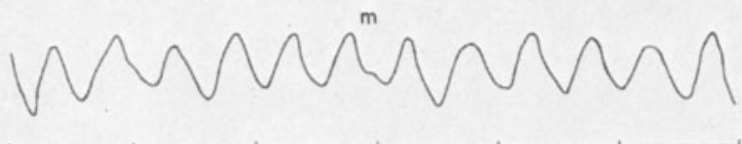

TIME (30 SEC. INTERVALS)

Fig. 1. X-ray photograph of cat stomach following a barium meal. The cat is supine. Figures $1 \mathrm{a}$ and $1 \mathrm{~b}$ show the electrical recordings obtained from the sites correspondingly marked, and Fig. $1 \mathrm{~m}$ shows the mechanical record from the strain gauge (site also marked). Distance between Sites a and b was $4 \mathrm{~cm}$, with reference electrode over left femoral muscle.

\section{METHOD}

Seventeen cats were used in preliminary studies to develop recording technique. Four additional animals, females ranging in weight from 2.2 to $3.3 \mathrm{~kg}$ were used to obtain the recordings analyzed below. All animals were wormed, distempered, and examined for general condition of health. All were maintained on a high-grade ad lib diet throughout the experimental period.

The present experiments used chronically implanted, extraluminal, contractile force transducers similar to those described by Jacoby, Bass and Bennett (1963). Baldwin-Lima-Hamilton Type SR-4, PEA-25-12 postyield strain gauges constituted the basic transducer unit. Polyethylene-coated No. 32 standard wire leads were soldered to each terminal, and the unit's surface coated on both sides with Dow Corning 732 RTV Silastic. The unit was sandwiched between two small silicone rubber sheets (Dow Corning Medical Grade Silastic), allowed to cure for $24 \mathrm{~h}$, and then tested electrically for reliability. Finally, two Amphenol, Type 220-804-2 female gold contacts were soldered to the free ends of the wire leads for later attachment to a connector mounted on the skull.

The sterilized transducer unit was sutured through the edges of its Silastic cover to the upper two smooth muscle layers at the site on the lower body of the stomach. Leads were then passed subdermally to a small dorsal incision in the skin over the skull and inserted into a Type 233, 5-contact Amphenol Micro-Med connector. The full assembly, which also contained a mounting for a cannula (see later) was attached to the skull with dental cement. During recording sessions, the force transducers were coupled to a dc Type $\mathrm{R}$ Offner dynograph through a strain gauge bridge circuit powered by a 6-V wet-cell battery (Stacy, 1960). The location of the transducer unit was later verified by X-ray photography. Sedatives were given to immobilize the animals during recordings of EGGs and mechanograms; the drugs were administered through an intraventricular cannula so as to minimize peripheral effects. The cannula was introduced into the right ventricle, using standard stereotaxic procedures. Postoperative health of the animals was excellent, and no obvious tissue reactions were apparent on autopsy. In one case, histological examination showed that smooth muscle had overgrown the entire transducer unit. Either standard EEG silver-silver chloride or suction-type electrodes were used with a saline electrode paste. The standard position for the active electrode was $2 \mathrm{~cm}$ caudal to the xiphoid process, but a second active electrode was used during many sessions to explore other locations on the abdomen. The reference electrode was always positioned on the skin over the left femoral muscle. The electrodes were direct coupled to a dc, Type $R$ Offner dynograph through a low-pass RC filter with a time constant of $2.5 \mathrm{sec}$, to eliminate EKG artifacts.

\section{Procedure}

Animals were given at least a week to recover from surgery before any attempt was made to obtain recordings. They were food-deprived for $24 \mathrm{~h}$ before the recording sessions, then fed ad lib on either raw beef or cheese to ensure good peristalsis during recording.

Chloralose (between 1.0 and $2.5 \mathrm{mg}$ ) in .5 -cc normal saline was administered via the cannula. It should be emphasized that chloralose was given only as a sedative to reduce movements. No discomfort was involved during recording, so that anaesthetic doses would have been inappropriate. Gastric motility, as measured via the force transducer, was depressed during the first $30 \mathrm{~min}$ after chloralose injection, while the sedative effect remained for up to $4 \mathrm{~h}$. Recording took place in a shielded cage, and began within $5 \mathrm{~min}$ of the electrodes being attached; it continued until effects of the sedative were reduced to the extent that the animal's movement produced artifacts in the tracings, a period, as noted, of up to $4 \mathrm{~h}$.

\section{RESULTS AND DISCUSSION}

\section{Analysis of Records}

Two methods were used in the analysis of records, samples of which are shown in Fig. 1. In the conventional analysis (Davis et al, 1957), tracings were visually examined for areas with a minimum number of movement artifacts and resets due to drift in the EGG. In the case of the slower waves, frequency was estimated by measuring the time in seconds for the occurrence of 10 successive waves, and converting to a rate-per-minute value. For faster activity, frequency was determined by counting the number of waves which occurred in the same time interval as the 10 slow waves, and again converting to a rate-per-minute value. This procedure was repeated for the corresponding mechanogram, using the exact time interval in which the 10 electrical waves occurred.

A second method was also used as an illustrative exercise. It involved the use of autocorrelation, an averaging process in which a segment of record is essentially slid over itself for a range of "lag points." Figure 2 shows the autocorrelation of the three records shown in Fig. 1. This averaging technique loses random 
noise from the immediate record, so that overall complexity is markedly reduced and interpretation becomes much easier. The random noise actually appears as slope between zero and the 150th lag point, so that the EGG at Location b can be seen to be much noisier than the EGG at Location a: the mechanical record, $m$, is almost noise free. A more detailed description of autocorrelation and its uses can be found in Rosenblith (1962); in addition, Small, Brean, and Farrar (1955) discuss the method in some deatil and attempt to apply it to balloon data from rabbit intestine.

The autotocorrelation can then be broken down into its various frequency components by fitting a Fourier series. This produces "power spectra" for the electrical and mechanical records, as in Fig. 3, where each abscissa shows the relative contribution of the various frequency components to the original record. Attinger, Anne, and McDonald (1966) have an excellent discussion of the uses and limitations of Fourier analysis in physiology and may be consulted for rationale and background.

Finally, crosscorrelations were computed as a means of determining relations between pairs of electrical and of mechanical and electrical tracings from the same cat.
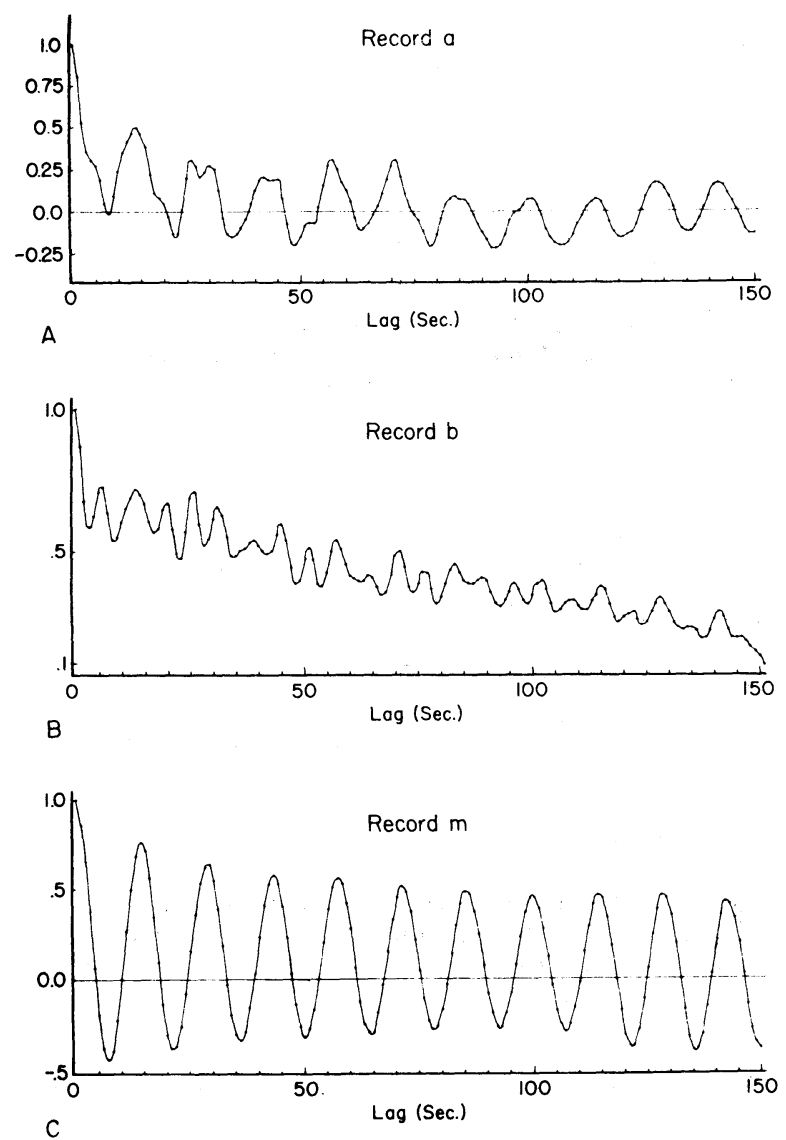

Fig. 2. Autocorrelations of the sample records in Figs. 1a, 1b, $1 \mathrm{~m}$. The abscissa represents time lag from 0 to $150 \mathrm{sec}$ in increments of $5 \mathrm{sec}$. The ordinate is a normalized relative scale and has no real meaning other than for comparison between correlograms.
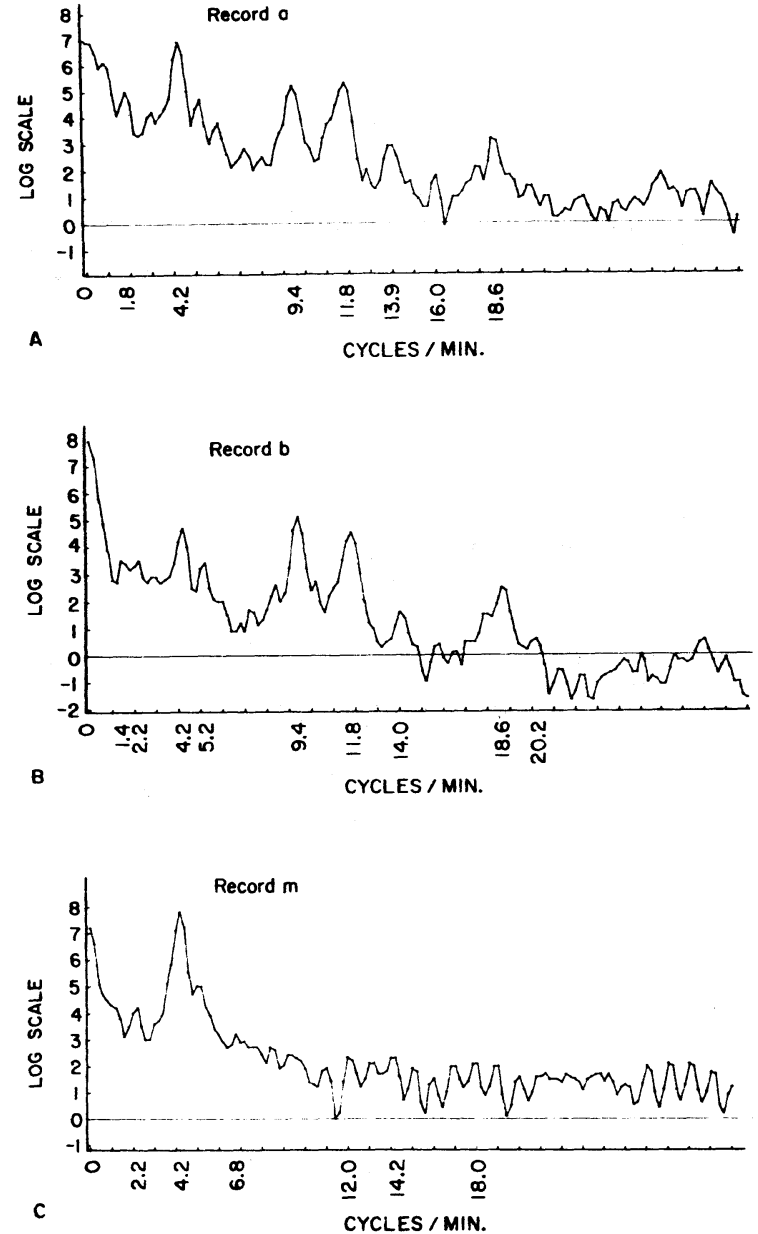

Fig. 3. Power spectra of autocorrelations shown in Fig. 2. The abscissa represents frequency, in $1.02-$ cycle/min divisions. Ordinate is again an arbitrary log power value.

This technique (Rosenblith, 1962) is analogous to autocorrelation except that the the values of the correlogram now arise from correlating two different records rather than a single record. These analyses make it possible to identify periodicities common to each pair of records, or "time series," and in addition to identify phase differences between them. If two records are in phase, a peak will occur at the zero lag point (Fig. 4, upper tracing), wheeras if they are out of phase (Fig. 4, middle and lower tracings), the degree to which they are out of phase appears in the correlogram. The analyses described above were carried out, using the UCLA Biomedical Program BMD02T. Features of the analysis and implications for interpretation are discussed in the following sections.

\section{Correlations Between EGG and Mechanogram}

A large volume of data was obtained, although at this experimental stage, much had to be discarded because of movement artifacts, weak force transducers, lack of significant gastric activity or other technical problems. Records were chosen for this paper on the basis of 

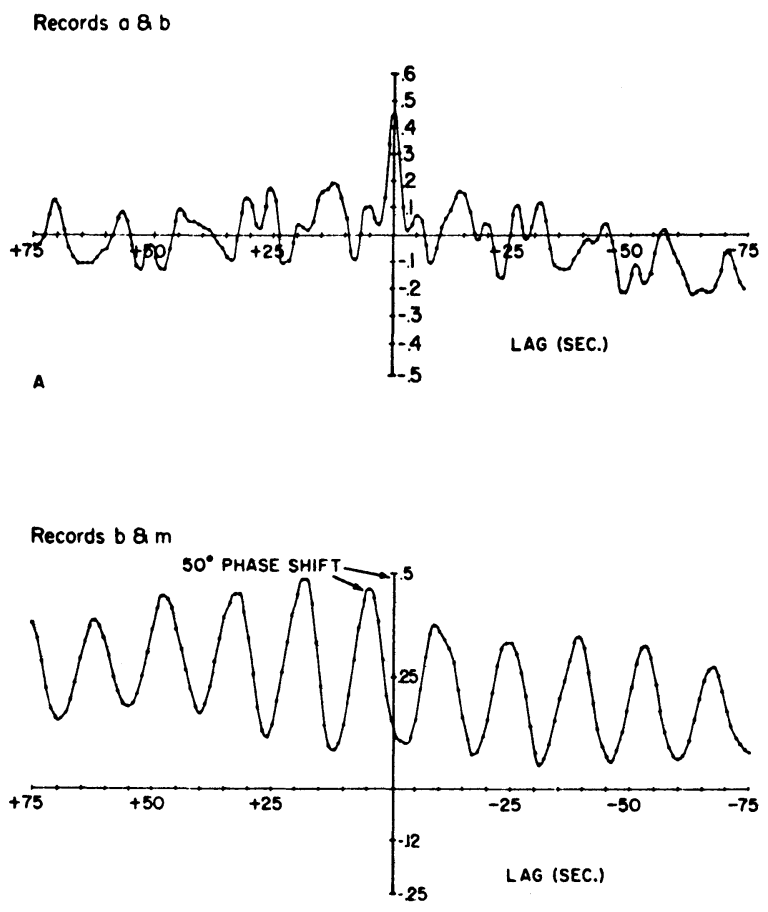

B

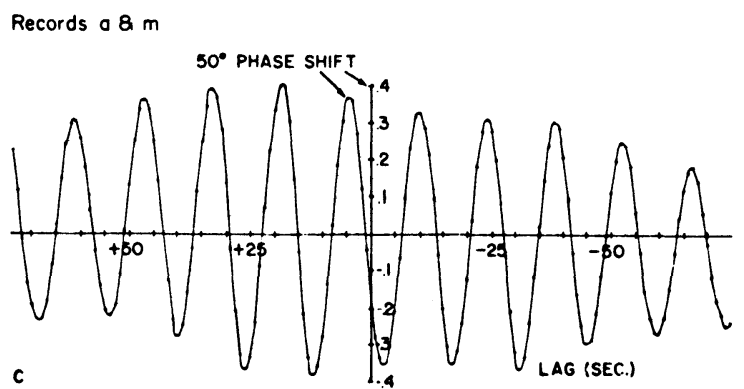

Fig. 4. Crosscorrelations of records in Fig. 1. Ordinate is a normalized relative scale as in autocorrelations, and the abscissa is time lag $\pm 75 \mathrm{sec}$. If two records are in phase, a peak occurs at the zero lag point as in A. Similarly, two records out of phase, as indicated in B and $C$, will have a corresponding phase shift from the zero lag.

having good activity and being artifact free, but were not otherwise "selected." Hand reading analysis is based on samples of 140, 340, and 100 slow waves for Cats 1,2, and 3 , respectively. The mean slow wave component in both electrical and mechanical records fell between 3.76 and 4.54 cycles/min for all three cats, and correlations between the two records are $.84, .86$, and .85 , all significant at $p<.005$. The mean fast wave electrical activity for the three cats was $12.9,11.2$, and 16.5 cycles/min, respectively; fast activity was not consistently readable in the mechanical record.

The close similarity of the electrical and mechanical records is especially evident in Fig. 6. This shows the effects of mechanically stretching the stomach by
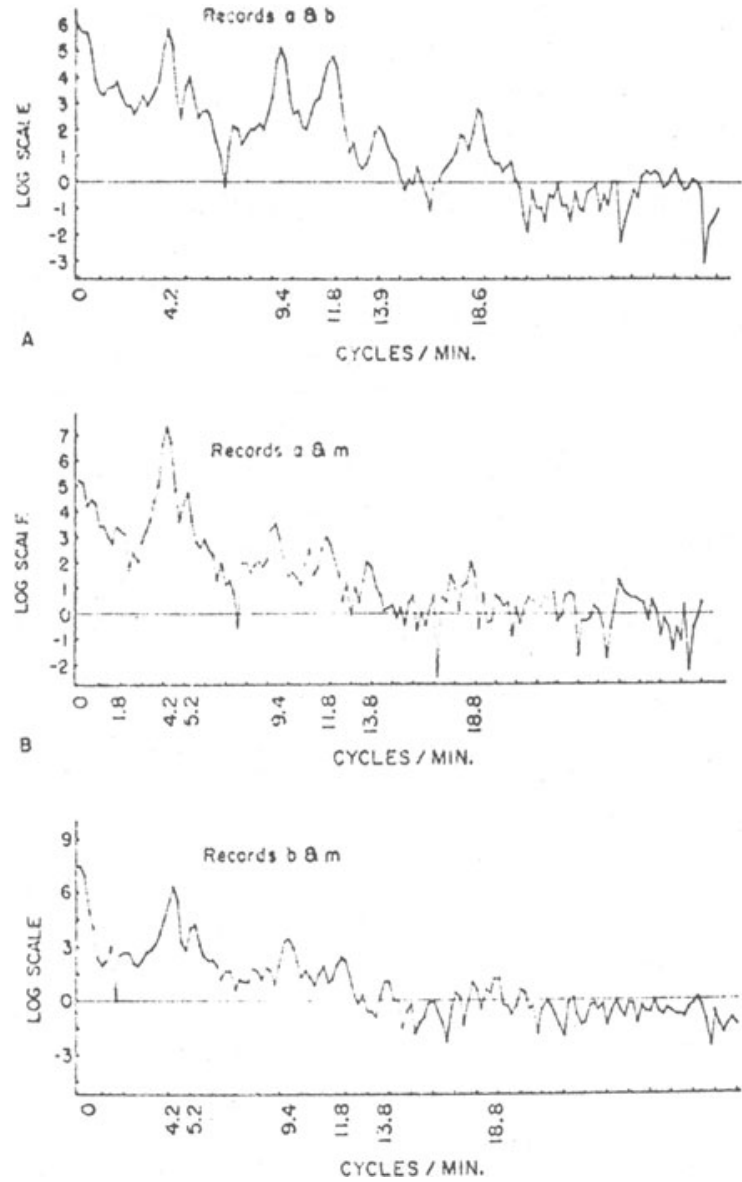

Fig. 5. Power spectra of crosscorrelations in Fig. 4. The ordinate is an arbitrary $\log$ power scale, and the abscissa is frequency in 1.02-cycle/min increments.

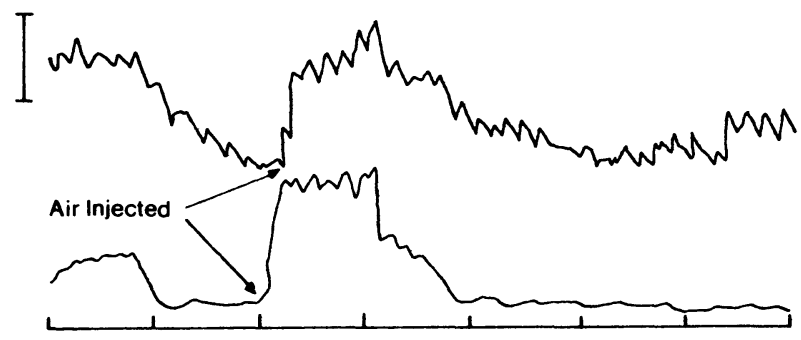

Voltage Bar $=2 \mathrm{mv}$

Time Base $=30 \mathrm{sec}$

Fig. 6. A simultaneous electrical and mechanical record showing the effects of increasing the air pressure inside the stomach. Lag between records is apparent only, due to curvilinear recording.

blowing air into the gastric lumen via an esophageal catheter. The tracings are seen to vary in a highly correlated manner, with all components appearing to be affected. The X-ray of this particular cat indicated that an intestinal segment was in proximity to the force transducer leading to the fast periodic activity found in both the EGG and mechanogram. 


\section{Computer Methods}

A 3-min segment of the 15-min recording subjected to computer analysis is shown in Fig. 1. Visual inspection suggests that each electrical record constitutes a complex biphasic time series, while the mechanogram has a base frequency of $4.1 \mathrm{cycles} / \mathrm{min}$, with a faster, 11-12-cycles/min component superimposed; the slower frequency is not as obvious in " $b$," and the faster component appears reduced in amplitude. The 4.1-cycle $/ \mathrm{min}$ wave completely dominates the mechanogram, "m," and looks to be out of phase with its corresponding component in "a."

When autocorrelation was applied to the sample record, the overall complexities of the EGG tracings were markedly reduced, as shown by the plots in Fig. 2 . Since Gaussian random noise is transformed to exponential form and appears as the slope of the composite curve between 0 and the 150th lag point, there is evidence that random noise was greater in Tracing " $b$ " than in Tracing "a," while in the mechanogram, tracing " $\mathrm{m}$ " is almost noise-free. With the data in this form, periodicities are much easier to identify and relations between tracings more readily apparent.

The fitting of a Fourier series to the data makes it possible to determine the relative frequencies at which the basic periodicities occurred in each sample of record. Figure 3 shows that in the two EGG tracings, the most prominent peaks occurred at five major frequencies: 4.2 , $9.4,11.8,13.9$, and $18.6 \mathrm{cycles} / \mathrm{min}$.

Crosscorrelations, computed for each pair of the sample records, are plotted in Fig. 4. The plots make it possible to identify periodicities common between pairs of records, the high degree of correspondence between the mechanogram and each of the EGGs being particularly striking. The occurrence of a peak at the zero lag point in the correlogram for Records "a" and " $b$ " indicates that there was no detectable phase shift between the two EGG tracings. This appeared to be true of all records regardless of the electrode position; however, as previously mentioned, this record was the only one subjected to computer analysis. In view of the fact that the two recording electrodes were located more than $4 \mathrm{~cm}$ apart, this observation deserves special attention in interpreting the results. Figure 4 shows clear phase shifts in the mechanical and electrical recordings, differences between the peak and zero lag points in the correlogram corresponding to a shift of $50 \mathrm{deg}$.

Fourier analysis provided data for relative power spectra plotted in Fig. 5 . The power spectrum between the two EGGs (upper trace) shows major peaks at the same five frequencies as those in the autocorrelation analysis (Fig. 3): 4.2, 9.4, 11.8, 13.9, and $18.6 \mathrm{cycles} / \mathrm{min}$. In the crosscorrelations between the EGGs and the mechanogram, the peak at 4.2 cycles/min is much more prominent in comparison with peaks at other frequencies.

Results of the present experiments indicate that the
EGG is, at the very least, correlated with gastric motility. Conventional analysis of the EGGs and the mechanograms show a dominant activity for all cats in the range, 3.5 to 5.5 waves $/ \mathrm{min}$, with high correlations between the two types of recordings. This relation appears with even greater clarity when the EGGs and mechanograms are subjected to the more sophisticated analysis of crosscorrelation and then fitted with a Fourier series. These results are consistent with reports of experiments involving recordings from electrodes placed directly on the surface of the stomach; for example, Daniel and Chapman (1963) have described activity in the range of 3 to 6 waves/min with electrodes on the antrum of the dog. Taken together, these and other sources of evidence (see Russell \& Stern, 1967) support the assertion that the EGG reflects the electrical and mechanical activity of the stomach.

It is apparent from the power spectra in Fig. 3 that the EGG as recorded by the Davis method also registers other rhythmic activity. This is in contrast to the mechanogram which normally had but one dominant peak frequency. Peaks in the EGG at 9.4, 11.8, 13.9, and 18.6 waves/min approximate the ranges of frequencies recorded by Daniel and Chapman (1963); Holaday, Volk, and Mandell (1958), and McCoy and Bass (1963) with electrodes placed directly on the ileum jejunum, and the duodenum, suggesting the possible sources of these four components.

Prosser and Bortoff (1968) have reviewed the literature concerning electrical activity of the intestine and stomach. They conclude that the slow waves probably do not directly indicate muscular contraction, but more likely facilitate spike synchronization and thus coordinate muscular activity. This assertion would seem compatible with the present findings which do not argue for a direct causational link between electrical and mechanical phenomena only for a strong covariance between the two. Furthermore, while the correlations are high at over .80 , they are not of course perfect, and we ourselves have observed during recording sessions fluctuations in the electrical record which appear to have no counterpart in the mechanical record.

The application of autocorrelation and Fourier analysis have been shown to yield information unattainable by the conventional method of analysis, but certain disadvantages should be noted. First, because of the averaging process it entails, the method can be used only for the general description of the EGG and not for changes which occur within a record. Analysis of shorter time segments would reduce this problem, but never completely; the problem also arises that, as the sample is shortened, the theoretical assumption of an infinite time series is violated to an increasingly greater extent. Secondly, data must be converted to digital form which, unless analog-to-digital equipment is available, is extremely tedious. Thirdly, the method of autocorrelation assumes that the data being analyzed are periodic and represent a stationary, stochastic time 
series. Farrar (1960) has found the method to be unreliable for any analysis of records obtained by intragastric balloon technique and has pointed out that such data do not satisfy the above assumptions. However, the biphasic EGG and mechanogram tracings recorded in the present experiments, although not completely stationary and periodic, approximate these assumptions so closely as to provide the consistent results reported earlier.

The present report is not intended to demonstrate that the mechanical and electrical records of the active stomach are exact mirror images of each other. Indeed, the general relationship between the two is fairly "loose-coupled" and, as noted earlier, one can find segments of record where slow wave electrical activity appears to occur in the presence of a quiet mechanical record. Nor is it suggested that autocorrelation and Fourier analysis are necessary for an effective use of the EGG: these techniques do remove random sources, but they also remove other aperiodic features which could, depending on circumstances, be of greater clinical or experimental significance than data from overall analysis. The aim is, rather, to show that the surface EGG is simple to record, that it provides a veridical picture of the electrical activity of the stomach and that this activity correlates well with a simultaneous mechanical record. On these grounds, the EGG would seem to constitute a valuable indicator whose use has been needlessly neglected in psychophysiology.

\section{REFERENCES}

Alvarez, W. C. The electrogastrogram and what it shows. Journal of the American Medical Association, 1922, 78, 1116-1119.
Attinger, E. O., Anne, A., \& McDonald, D. A. Use of Fourier series for the analysis of biological systems. Biophysical Journal, 1966, 6, 291-304.

Daniel, E. E., \& Chapman, K. M. Electrical activity of the gastrointestinal tract as an indication of mechanical activity. American Journal of Digestive Diseases, 1963, 8, 54-102.

Davis, R. C., Garafolo, L., \& Gault, F. P. An exploration of abdominal potentials. Journal of Comparative \& Physiological Psychology, 1957, 50, 519-523.

Farrar, J. T. Use of digital computer in the analysis of intestinal motility. I.R.E. Transactions on Medical Electronics, 1960, 7, 259-263.

Goodman, E. N., Sandler, B., \& Sullivan, M. A probe for measuring the electrical activity of the human stomach. American Journal of Gastroenterology, 1964, 42, 511-514.

Holaday, D. A., Volk, H., \& Mandell, J. Electrical activity of small intestine with special reference to origin of rhythmicity. American Journal of Physiology, 1958, 195, 505-515.

Jacoby, H. I., Bass, P., \& Bennett, D. R. In vivo extraluminal contractile force transducer for gastro-intestinal muscle. Journal of Applied Physiology, 1963, 18, 658-665.

Krasil'nikov, L. G. Interpretation of electrogastrogram and its variants in healthy persons during digestion. Federation Proceedings (Trans. Supplement), 1964, 23, 901-904.

McCoy, E. J., \& Bass, P. Chronic electrical activity of gastroduodenal area: Effects of food and certain catecholamines. American Journal of Physiology, 1963, 205, 439-445.

Prosser, C. L., \& Bortoff, A. Electrical activity of intestinal muscle under in vitro conditions. In C. F. Code (Ed.) Handbook of Physiology. Baltimore: Williams \& Wilkins, Vol. IV. 1968 .

Kosenblith, W. A. Processing neuroelectrical data. Cambridge, Massachusetts: M.I.T. Press, 1962.

Russell, R. W., \& Stern, R. M. Gastric motility: The electrogastrogram. In P. Venables and J. Martin (Eds.), Manual of psychophysiological methods. Amsterdam: North Holland, 1966. Pp. 221-243.

Small, M. D., Brean, J. W., \& Farrar, J. T. An application of autocorrelation methods to the interpretation of intestinal motility records. Journal of General Physiology, 1955, 38 695-707.

Stacy, W. R. Biological and medical electronics. New York: McGraw-Hill, 1960
(Received for publication August 24, 1973;
revision received February $19,1974$. ) 